\title{
Numerical analysis and experimental research on load carrying capacity of water-lubricated tilting-pad thrust bearings
}

\author{
Xiuli Zhang ${ }^{1,2}$, Gengyuan $\mathrm{Gao}^{2,}{ }^{*}$, Zhongwei Yin ${ }^{2}$, Yanzhen Wang ${ }^{2}$, and Chao Gao ${ }^{2}$ \\ ${ }^{1}$ School of Mechanical Engineering, Shandong University of Technology, Zibo, PR China \\ 2 State Key Laboratory of Mechanical System and Vibration, Shanghai Jiao Tong University, Shanghai, PR China
}

Received: 10 August 2017 / Accepted: 10 January 2018

\begin{abstract}
Water-lubricated bearings are expected to be widely used because of convenience, green, safe and energy saving. The purpose of this study is to investigate the load carrying property of water-lubricated tiltingpad thrust bearings. A large amount of numerical analyses are undertaken based on computational fluid dynamics and the optimization method of pivot location and the calculation method of minimum film thickness are summarized. A thrust bearing is designed according to the numerical results and is tested by experiments. The experimental results validate the numerical method and the minimum film thickness to surface roughness ratio corresponding to the change of bearing lubrication regime from mixed lubrication to hydrodynamic lubrication is obtained.
\end{abstract}

Keywords: Tilting-pad thrust bearing / water lubrication / CFD / experiment

\section{Introduction}

In turbomachinery, hydrodynamic thrust bearing is usually used to balance axial load. It plays an important role in movement security; its failing could involve serious damage following the possible contact between the rotor and the stator. Correct choice of the bearing type and prediction of its performance are therefore of great importance for trouble free machine operation [1]. Tilting-pad thrust bearing has the advantage of excellent stability, superior durability and high load carrying capacity. Thus this study focuses on the tilting-pad thrust bearing.

Presently the conventional lubricant is oil, but it has many disadvantages such as serious waste of resources, environmental pollution caused by oil leakage, explosive hazard, large bulk and complex structure, poor flexibility and maintainability [2]. In some conditions such as nuclear pumps, hydroturbine, marine pumps and integrated motor propulsors, water-lubrication replaces oil-lubrication. Heretofore, researches on water-lubricated bearings have focused primarily on the friction and wear characteristics of different bearing materials under water lubrication [2-6], and the lubrication performance of water-lubricated journal bearings [7-16], only a few of them are about water-lubricated thrust bearings. Zhang et al. [17] gave a brief summary of the influence factors of bearing capacity

\footnotetext{
* e-mail: gaoqi_1118@163.com
}

of water-lubricated thrust bearing and they pointed out that in order to guarantee the normal work of the bearing, the bearing capacity should be improved by changing factors including material of bearing and shaft, roughness of grinding area, bearing structure, pad number, pivot location, speed and the medium temperature, etc. Zhang et al. [18] studied the load carrying property of hydrodynamic water-lubricated step thrust bearing using CFD method and proposed the design method of this kind of bearing. Nakano et al. [19] developed a prototype of the next-generation, high-performance microturbine system using water-lubricated journal and thrust bearings and experimental results showed that the rotor system achieved stable rotating conditions at a rated rotational speed of $51000 \mathrm{rpm}$. Kansai and Hitachi Mitsubishi Hydro developed the water-lubricated tilting-pad thrust bearing for a vertical type hydraulic turbine generator. They studied the effect of support structure on the bearing film thickness by experiments and found that the offset support structure is much more stable than the center support structure [20].

Water-lubricated bearing has advantages of convenience, green, safe and energy saving [21], but its load carrying capacity is much smaller than that of oillubricated bearings. Works on water-lubricated bearings $[4,22-24]$ showed that the bearings usually operate in the regime of mixed lubrication and the lubrication mode might convert to hydrodynamic when load decreases. Wang et al. [22,23] studied the critical load, considering the sudden increase in the friction coefficient as the transition 


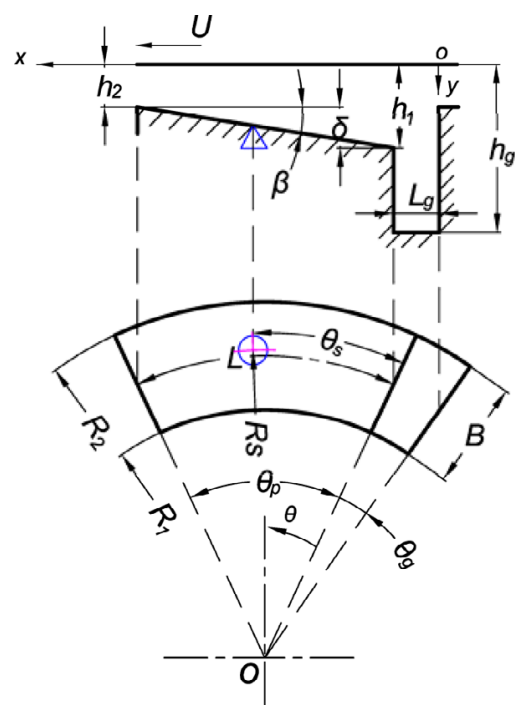

Fig. 1. Flow model of one sector pad and one groove of tiltingpad thrust bearing (not to scale).

of lubrication mode. In order to ensure that waterlubricated tilting-pad bearing works under hydrodynamic lubrication as far as possible, the present work first studies the design method of pivot location and the calculation method of minimum film thickness by numerical analysis, and then studies the minimum film thickness to surface roughness ratio corresponding to the change of bearing lubrication regime from mixed lubrication to hydrodynamic lubrication by experiments.

\section{Tilting-pad thrust bearing model}

Tilting-pad thrust bearing can be studied using taper-faced thrust bearing model. The difference is that pressure distribution on tilting pad should meet moment balance on the pivot. The supporting position of tilting-pad thrust bearing is the pressure center of taper-faced thrust bearing model. The previous researches on thrust bearing usually simplified the sector pad to rectangular pad and ignored the groove. In this work, the flow model of one sector pad and one groove of tilting-pad thrust bearing as shown in Figure 1 is studied. The thrust bearing is submersed in water. The hydrodynamic action generates dynamic pressure in water, primarily in the convergent part of the thrust pad, to counteract the load thereby separating the ring surface from the bearing surface with a thin lubricant film. Geometry parameters - inner radius $R_{1}$, outer radius $R_{2}$, number of pads $n$, pad ratio $k$, and pad supporting position- all influence the load carrying capacity of tilting-pad thrust bearings. In this work, the taper-faced thrust bearing models with different radii and pad inclined angles are calculated to study the effect of geometry parameters on load carrying capacity and supporting position of tilting-pad thrust bearing. According to the design of oil-lubricated thrust bearings [25], $R_{2}$ is usually 1.5-3 times of $R_{1}$. The number of pads $n$ is generally $6-12$. The pad ratio $k$, the percentage of pad area in the whole thrust surface, is typically $0.7-0.85$. Pad width-tolength ratio $B / L$ is a pad parameter determined by $R_{2} / R_{1}$, $n$ and $k$, and the relation is

$$
\frac{B}{L}=\frac{n}{\pi k} \cdot \frac{R_{2} / R_{1}-1}{R_{2} / R_{1}+1} .
$$

It has an effect on the optimum value of pad inclined angle $\beta$ and pad supporting position $R_{s}$ and $\theta_{s}$.

Rotational speed $N$, fluid viscosity $\mu$ and the minimum film thickness $h_{2}$ determine the bearing carrying performance as well. Rotational speed and fluid viscosity are determined by work conditions. The minimum film thickness should not be less than a safety value depending on surface roughness and system vibration, or the lubrication model might change from full dynamic lubrication to boundary or mixed lubrication.

\section{Numerical analysis}

\subsection{Governing equations}

The flow is considered laminar, steady and incompressible, with zero gravitational and other external body forces. The bearing is fully submerged into water. As water has a low viscosity (less than $0.001 \mathrm{~Pa} . \mathrm{s}$ ) and a large specific heat $(4200 \mathrm{~J} /(\mathrm{kg} . \mathrm{K}))$, the temperature rise of water-lubricated bearing is small. Thus the flow is considered isothermal. To solve such flow, the following governing equations must be solved:

Mass conservation equation

$$
\nabla \cdot \mathbf{v}=0 .
$$

Momentum conservation equations

$$
\rho(\mathbf{v} \cdot \nabla) \mathbf{v}=-\nabla p+\mu \cdot \nabla^{2} \mathbf{v} .
$$

When flow enters the groove, pressure might fall below the saturation water vapor pressure, and the liquid would rupture and cavitation occurs. Thus cavitation is taken into account. In the present work the CFD code ANSYS FLUENT is used. There are three available cavitation models in ANSYS FLUENT: Singhal et al. model, ZwartGerber-Belamri model and Schnerr and Sauer model. The Singhal et al. model is numerically less stable and more difficult to use. The Zwart-Gerber-Belamri and the Schnerr and Sauer models are robust and converge quickly [26]. In this case the Zwart-Gerber-Belamri model is employed.

In cavitation, the liquid-vapor mass transfer (evaporation and condensation) is governed by the vapor transport equation [26]:

$$
\frac{\partial}{\partial t}\left(\alpha_{v} \rho_{v}\right)+\nabla \cdot\left(\alpha_{v} \rho_{v} \mathrm{v}\right)=R_{g}-R_{c}
$$

where $R_{g}$ and $R_{c}$ account for the mass transfer source terms connected to the growth and collapse of the vapor bubbles, $\alpha_{v}$ is vapor volume fraction and $\rho_{v}$ is vapor density. In Zwart-Gerber-Belamri model, $R_{g}$ and $R_{c}$ are defined as follows[27]: 


$$
\begin{aligned}
& \text { If } p \leq p_{v} \quad R_{g}=F_{\text {evap }} \frac{3 \alpha_{\mathrm{nuc}}\left(1-\alpha_{v}\right) \rho_{v}}{R_{b}} \sqrt{\frac{2}{3} \frac{p_{v}-p}{\rho_{l}}} \\
& \text { If } p \geq p_{v} \quad R_{c}=F_{\text {cond }} \frac{3 \alpha_{v} \rho_{v}}{R_{b}} \sqrt{\frac{2 p-p_{v}}{3}}
\end{aligned},
$$

where $F_{\text {evap }}=$ evaporation coefficient $=50, F_{\text {cond }}=$ condensation coefficient $=0.01, \quad R_{b}=$ bubble radius $=10^{-6} \mathrm{~m}$, $\alpha_{\text {nuc }}=$ nucleation site volume fraction $=5 \times 10^{-4}, \rho_{l}=$ liquid density, $p_{v}=$ pressure of vapor.

Supposed that there's no installation error and the load of a thrust bearing is distributed uniformly over all pads, the load and friction torque of a thrust bearing can be calculated by integrating the pressure and shear stress over the rotating wall as follows:

$$
F_{z}=n \int_{R_{1}}^{R_{2}} \int_{-\theta_{g}}^{\theta_{p}} p r d \theta d r, M_{\mathrm{fr}}=n \int_{R_{1}}^{R_{2}} \int_{-\theta_{g}}^{\theta_{p}} \tau r^{2} d \theta d r .
$$

The friction coefficient is

$$
f=\frac{M_{f r}}{F_{z} R_{m}} .
$$

The supporting position $\left(R_{s}, \theta_{s}\right)$ of tilting-pad thrust bearing meets the following formula

$$
\begin{aligned}
& \int_{R_{1}}^{R_{2}} \int_{0}^{\theta_{p}}\left(r-R_{s}\right) p r d \theta d r=0 \\
& \int_{R_{1}}^{R_{2}} \int_{0}^{\theta_{p}} r \sin \left(\theta-\theta_{s}\right) p r d \theta d r=0
\end{aligned} .
$$

To compare with results in the literature, nondimensional pressure, non-dimensional load carrying capacity and non-dimensional friction torque are defined as

$$
\begin{aligned}
p^{*} & =\frac{p h_{2}^{2}}{\mu U_{m} L}, F_{z}{ }^{*}=\frac{F_{z} h_{2}^{2}}{n \mu U_{m} L^{2} B}, M_{\mathrm{fr}}{ }^{*} \\
& =\frac{M_{f r} h_{2}}{n \mu U_{m} L B R_{m}} .
\end{aligned}
$$

The non-dimensional supporting position is defined as

$$
R_{s}^{*}=\frac{\left(R_{s}-R_{1}\right)}{B}, \theta_{s}^{*}=\frac{\theta_{s}}{\theta_{p}} .
$$

\subsection{CFD models}

The flow model is built and meshed in Gambit 2.3. The boundary condition is set as shown in Figure 2. Rotational periodic boundary condition is used to simplify the flow model and to reduce the computational cost. The operating pressure is set to $101325 \mathrm{~Pa}$. Since the bearing is fully submerged, the pressure at the inlet and outlet boundaries is taken as zero (gauge pressure). A no-slip condition is imposed on the solid walls. The bottom wall is stationary and the upper one is assumed to be rotating at a constant rotational speed $N$.

In the present study, water properties at $20^{\circ} \mathrm{C}$ listed in Table 1 are employed. In all cases, the groove depth $h_{\mathrm{g}}=2 \mathrm{~mm}$ and pad ratio $k=0.75$. For the purpose of finding

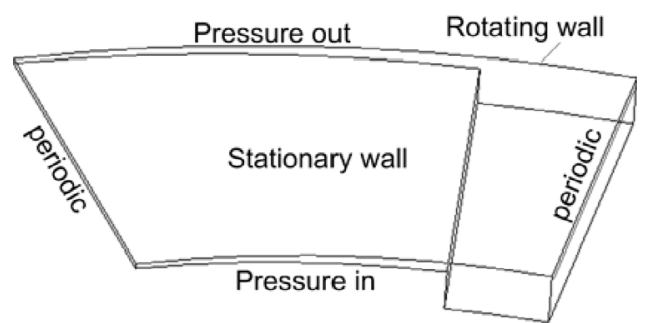

Fig. 2. Boundary conditions.

Table 1. Water properties at $20^{\circ} \mathrm{C}$.
Saturation water vapor pressure

Saturation density of water

Saturation density of water vapor

Dynamic viscosity of water

Dynamic viscosity of water vapor
$2340 \mathrm{~Pa}$

$998.2 \mathrm{~kg} / \mathrm{m}^{3}$

$0.5542 \mathrm{~kg} / \mathrm{m}^{3}$

$10^{-3} \mathrm{Pas}$

$1.34 \times 10^{-5} \mathrm{Pas}$ relations between load carrying properties and pad parameters, the inner radius $R_{1}$ is set to $30 \mathrm{~mm}$, number of pad $n$ is 12 , rotational speed $N$ is $3000 \mathrm{rpm}, R_{2}$ varies from 35 to $70 \mathrm{~mm}$ and $\delta^{*}$ varies from 0.4 to 3 . The nondimensional inclined plane height $\delta^{*}$ varies to seek out the optimum ones. The outer radius, $R_{2}$, changes to find the effect of $B / L$ on the non-dimensional load $F_{z}{ }^{*}$, the optimum $\delta^{*}$ and the optimum supporting position. Pad width-tolength ratio $B / L$ can be calculated from equation (1).

Due to the existence of the thin film in hydrodynamic bearings, the dominating feature for bearing CFD models is the large aspect ratio of the grid which is $330-4500$ in this study. However, the suggested value of aspect ratio is less than 100 or 200 for normal analysis. Thus double precision calculations are employed to avoid the negative influence of the large aspect ratio according to ANSYS FLUENT user manual [26]. The CFD models are meshed using hexahedron grids in Gambit 2.3. In order to obtain accurate solutions, a mesh refinement study is carried out. Since the groove area contributes little to the load carrying capacity, the groove length $L_{\mathrm{g}}$ is divided into 15 cells and the groove height $h_{\mathrm{g}}$ is divided into 20 cells for all cases. An expansion ratio of 1.4 is employed in $h_{\mathrm{g}}$ to avoid sudden change of mesh density near the thin film thickness. A uniform mesh is employed in other edges of the model. The influence of the mesh density of $h_{1}, L, B$ on load for the bearing model with $R_{1}=30 \mathrm{~mm}, \quad R_{2}=45 \mathrm{~mm}, \quad n=12, \quad h_{2}=10 \mu \mathrm{m}$, $\beta=0.056^{\circ}, N=3000 \mathrm{rpm}$ is studied and the results are summarized in Table 2. As cell number of $h_{1}$ increases, computed result increases. As cell number of $L(B)$ increases, computed result decreases. Based on the result, case 8 is employed in this study. Appropriate mesh sizes are also found for other sizes of bearing models.

\subsection{Results and discussion}

\subsubsection{Pressure distribution}

Figures $3 \mathrm{a}-\mathrm{c}$ present the gauge pressure distribution of tilting-pad thrust bearing for different inclined angles, and $D_{i}=60 \mathrm{~mm}, D_{o}=90 \mathrm{~mm}, h_{2}=10 \mu \mathrm{m}, N=3000 \mathrm{rpm}$. The 
Table 2. Mesh refinement study.

\begin{tabular}{|c|c|c|c|c|c|}
\hline \multirow[t]{2}{*}{ Case } & \multicolumn{3}{|c|}{ Number of cells } & \multirow[t]{2}{*}{ Number of hexahedral elements } & \multirow[t]{2}{*}{$\mathrm{F}_{\mathrm{z}}(\mathrm{N})$} \\
\hline & $h_{1}\left(h_{2}\right)$ & $L$ & $B$ & & \\
\hline 1 & 6 & 45 & 45 & 16500 & 356.09 \\
\hline 2 & 8 & 45 & 45 & 21900 & 358.01 \\
\hline 3 & 10 & 45 & 45 & 27300 & 358.84 \\
\hline 4 & 12 & 45 & 45 & 32700 & 359.05 \\
\hline 5 & 10 & 15 & 15 & 4800 & 361.80 \\
\hline 6 & 10 & 30 & 30 & 13800 & 360.24 \\
\hline 7 & 10 & 45 & 45 & 27300 & 358.84 \\
\hline 8 & 10 & 60 & 60 & 45300 & 358.31 \\
\hline 9 & 10 & 100 & 100 & 115300 & 357.98 \\
\hline
\end{tabular}

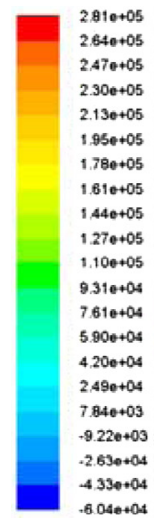

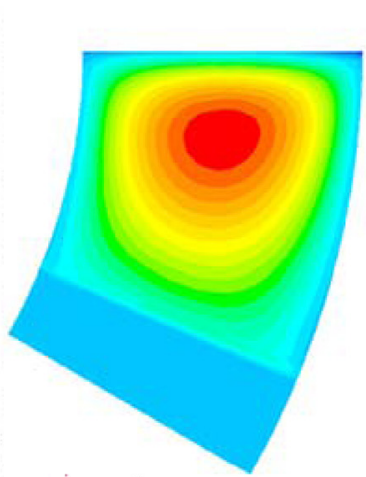

(a) $\delta^{*}=1$

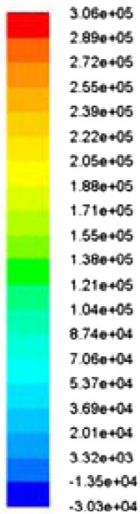

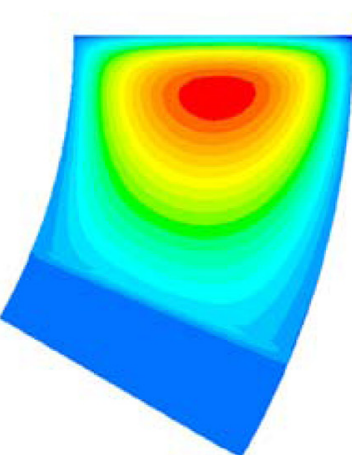

(b) $\delta^{*}=2$

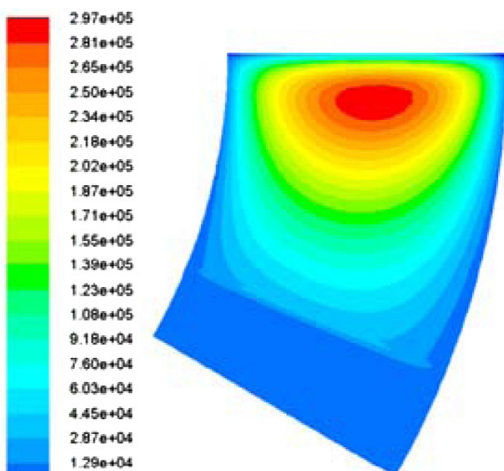

(c) $\delta^{\mathrm{k}}=3$

Fig. 3. Pressure distribution for pads with different $\delta^{*}$, and $B / L=1.02, h_{2}=10 \mu \mathrm{m}, N=3000 \mathrm{rpm}$.

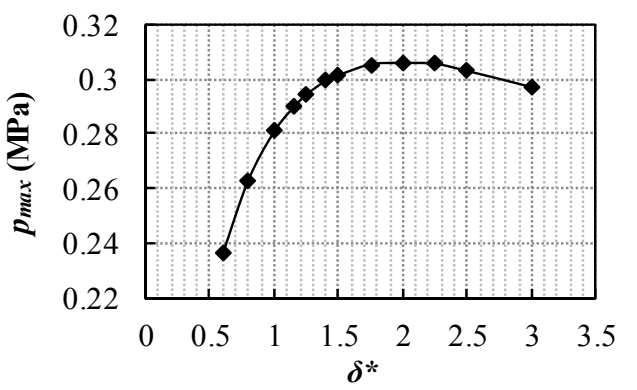

Fig. 4. Maximum pressure, $p_{\max }$, versus $\delta^{*}$, and $B / L=1.02$, $h_{2}=10 \mu \mathrm{m}, N=3000 \mathrm{rpm}$.

pressure distribution shows that the maximum pressure of the whole film is located near the outlet. Figure 4 shows the maximum pressure versus non-dimensional height of inclined plane, $\delta^{*}$. It shows that as $\delta$ increases, the pad inclined angle increases and the maximum pressure first increases and then decreases. The maximum pressure reached the maximum value at $\delta^{*}=2$, but the effective load region size decreases. So there is an optimum $\delta^{*}$ to get the maximum load carrying capacity. When the fluid flows into the groove, pressure on the stationary wall drops rapidly, which might cause cavitation if it is less than $2340 \mathrm{~Pa}$. As the fluid velocity near the outer radius is larger than that near the inner radius, the pressure drop is more significant and cavitation occurs more easily. From the CFD analyses, it is found that the cavitation zone will get larger when the minimum film thickness decreases or the fluid velocity increases.

Figure 5 compares the pressure distribution of tiltingpad thrust bearing for different load and rotational speed, and $D_{i}=60 \mathrm{~mm}, D_{o}=90 \mathrm{~mm}, R_{s}{ }^{*}=0.51, \theta_{s}{ }^{*}=0.575$. From Figure $5 \mathrm{a}, \mathrm{b}$, it can be seen that when the load is constant, the pressure distribution changes little with the rotational speed, but the minimum film thickness increases as the rotational speed increases. From Figure 5b, c, it can be seen that when the rotational speed is constant, the pressure increases and the minimum film thickness decreases as load increases.

Figure 6a, b show the non-dimensional pressure distribution at the radius of maximum pressure and at the medium radius of tilting-pad thrust bearing for different $B / L$ respectively, and $\delta^{*}=1, \quad h_{2}=10 \mu \mathrm{m}$, $N=3000 \mathrm{rpm}$. The non-dimensional pressure distribution of infinite width slider bearing in [28] is shown for comparison. Figure 6 a indicates that the maximum $p^{*}$ at the radius of maximum pressure increases as $B / L$ increases, and it increase sharply when $B / L$ is less than 1.5 , but increase slowly when $B / L$ is more than 1.5 . Figure $6 \mathrm{~b}$ shows 

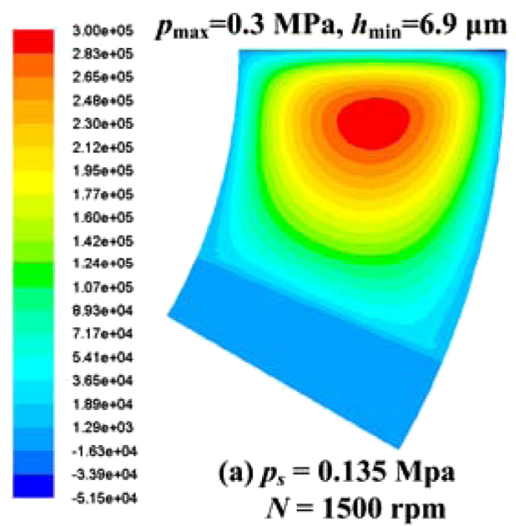
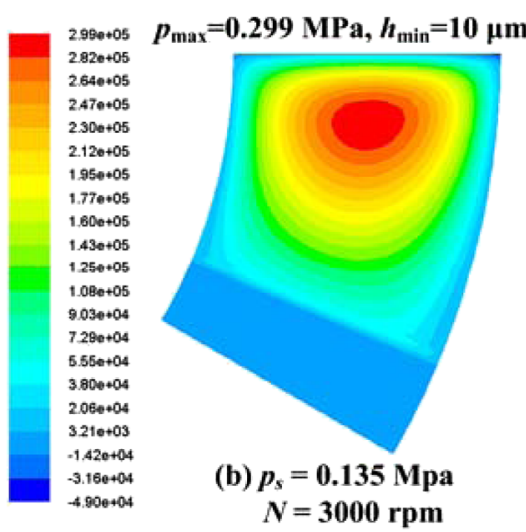
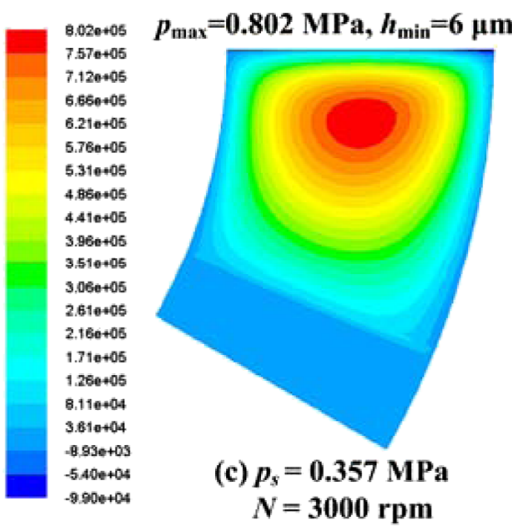

Fig. 5. Pressure distribution for different load and rotational speed.
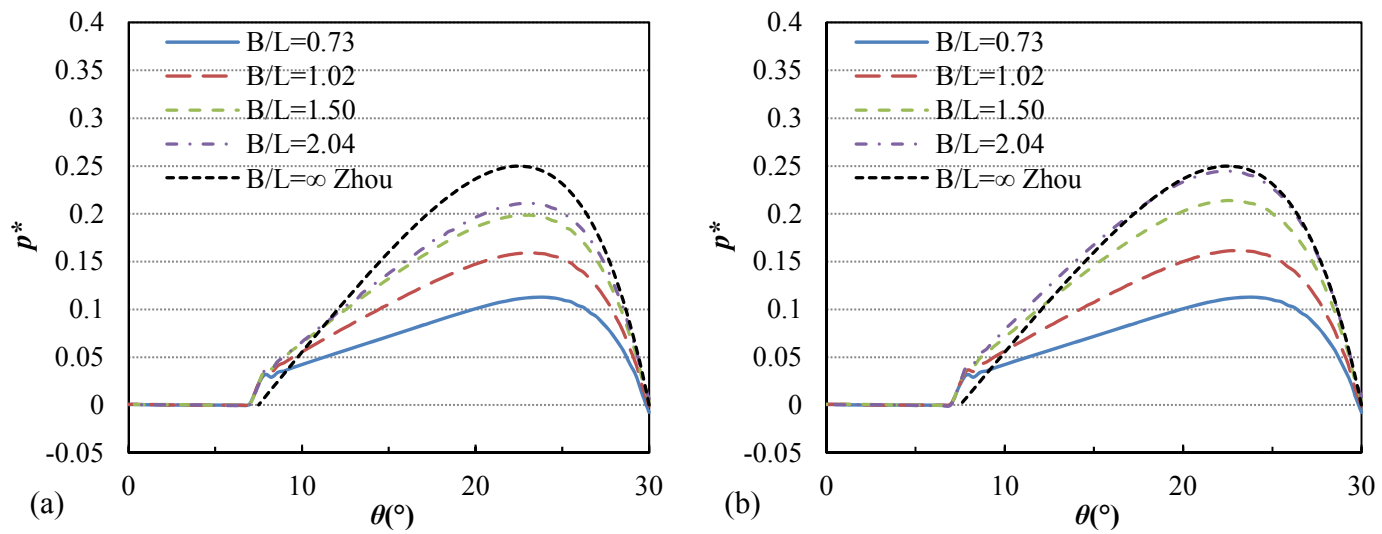

Fig. 6. Non-dimensional pressure distribution at (a) the radius of maximum pressure, (b) the medium radius of tilting-pad thrust bearing for different $B / L$, and $\delta^{*}=1, h_{2}=10 \mu \mathrm{m}, N=3000 \mathrm{rpm}$.

that when $B / L$ is more than 1.5 , non-dimensional pressure at the medium radius is larger than that at the radius of maximum pressure, and the non-dimensional pressure distribution at the medium radius for $B / L=2.04$ is close to that of infinite width slider bearing. This can be explained by the inertial effect of sector pad thrust bearing. The maximum pressure moves outwards due to the inertial effect, and it reacts on the pressure buildup at the medium radius and increases $p_{\max }{ }^{*}$ at the medium radius.

\subsubsection{Optimization of load carrying capacity}

Figure 7 presents the non-dimensional load carrying capacity $F_{z}{ }^{*}$ versus height of inclined plane $\delta$ and the non-dimensional height of inclined plane $\delta^{*}$ for different $h_{2}$ with $B / L=1.02$. Results illustrate that although the optimum $\delta$ is different for different minimum film thicknesses, the optimum ratio of them, the non-dimensional height of inclined plane $\delta^{*}$, is always around 1.33. Figure 8 compares the optimum $\delta^{*}$ for pads with different $B / L$ values. It can be seen that the optimum $\delta$ is 1.4 for $B$ $L=0.73$ and the range of $\delta^{*}$ corresponding to more than 0.9 times the maximum $F_{z}^{*}$ is $[0.65,2.6]$; the optimum $\delta^{*}$ is 1.26 for $B / L=1.27$ and the range of $\delta *$ corresponding to more than 0.9 times the maximum $F_{z}{ }^{*}$ is $[0.62,2.42]$; the optimum $\delta^{*}$ is 1.2 for $B / L=2.04$ and the range of $\delta^{*}$ corresponding to more than 0.9 times the maximum $F_{z}{ }^{*}$ is $[0.6,2.3]$. The optimum $\delta^{*}$ is summarized in Figure 9. The wider the pad, the smaller the optimum $\delta^{*}$. The pad inclined angle can be calculated using the following equation:

$$
\beta=\frac{\delta_{o p t}^{*} h_{2}}{R_{m} \sin \theta_{p}} .
$$

\subsubsection{Optimization of pivot position}

Pivot position need to be chosen appropriately when designing tilting-pad thrust bearing. For tilting-pad thrust bearing supported by ball, the pressure distribution on pad surface should meet moment balance equations on the pivot, so the pressure center is the supporting position. Figures 10 and 11 present the non-dimensional radius, $R_{s}^{*}$, and non-dimensional angle, $\theta_{s}{ }^{*}$, of pivot versus $\delta^{*}$ for pads with different $B / L$ values. Figure 10 shows that $R_{s}^{*}$ is greatly influenced by $B / L$ and $R_{s}^{*}$ increases as $B / L$ increases; $R_{s}^{*}$ is less affected by $\delta^{*}$ and $R_{s}^{*}$ decreases as $\delta^{*}$ increases. Figure 11 shows that $\theta_{s}^{*}$ is greatly influenced by $\delta^{*}$ and $\theta_{s}^{*}$ increases as $\delta^{*}$ increases; $\theta_{s}^{*}$ is less affected by $B / L$ and the variation of $\theta_{s}^{*}$ decreases as $B / L$ increases. Figure 12 shows the change of pad pivot position with $B / L$ and $\delta^{*}$ in a two-dimensional coordinates. 

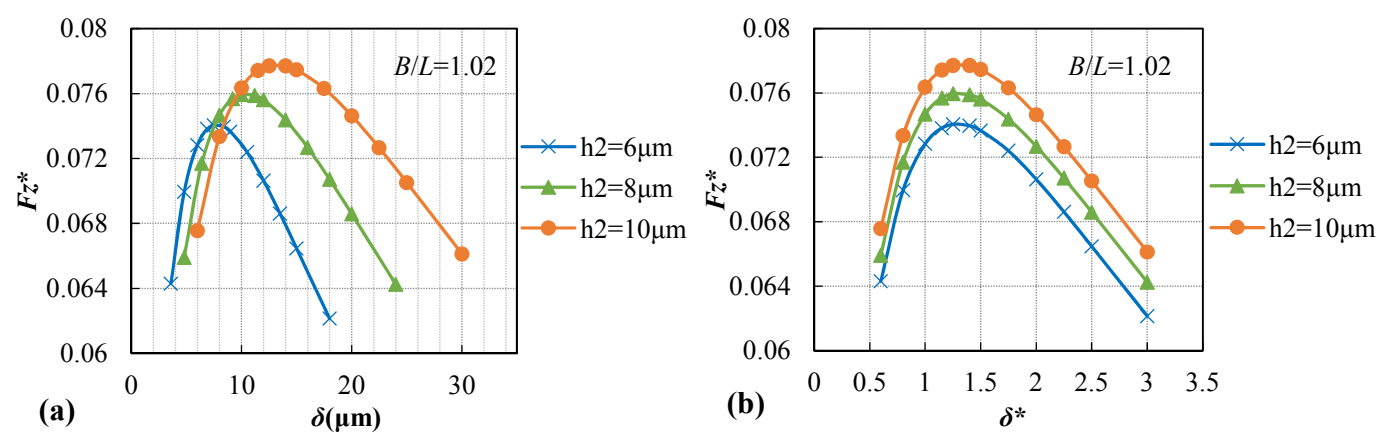

Fig. 7. Non-dimensional load carrying capacity, $F_{z}^{*}$, versus (a) height of inclined plane, $\delta$, and (b) height of inclined plane to minimum film thickness ratio, $\delta^{*}$, for different minimum film thicknesses, $h_{2}$, and $B / L=1.02$.
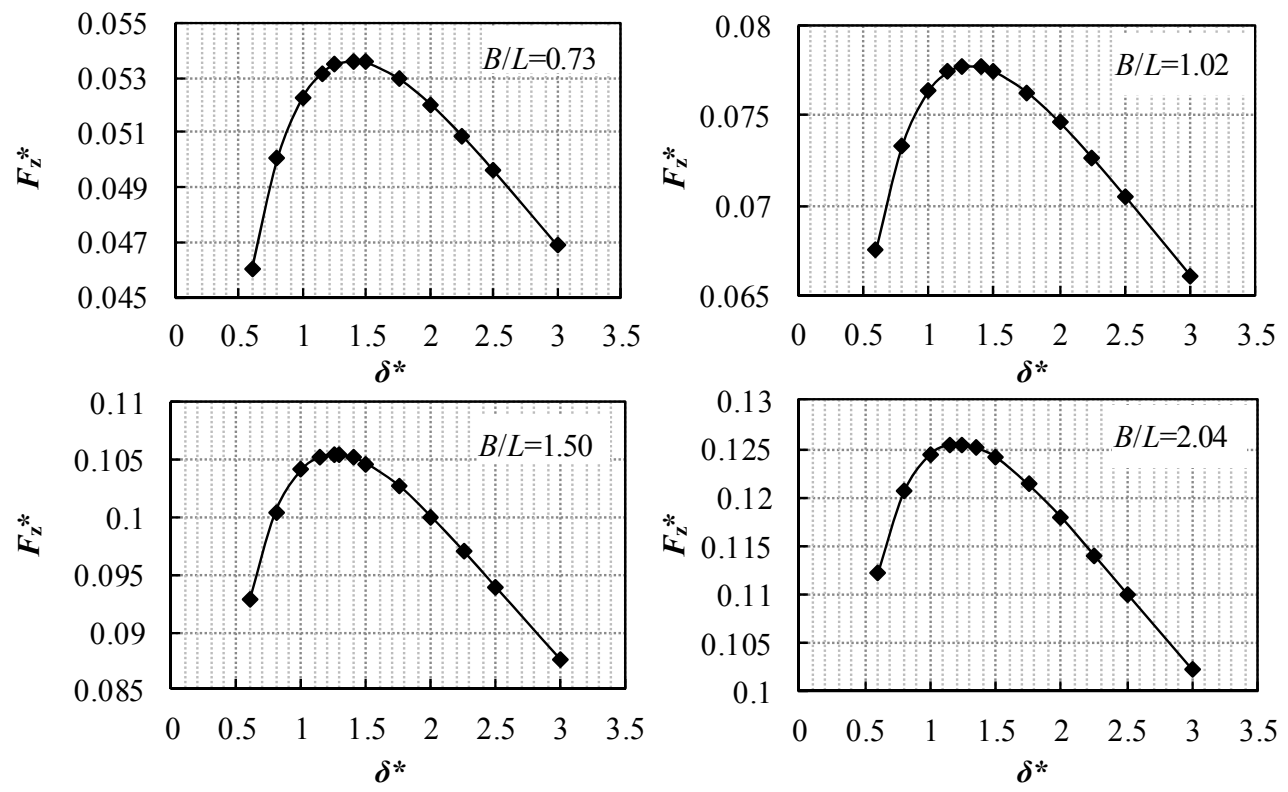

Fig. 8. Non-dimensional load carrying capacity, $F_{z}{ }^{*}$, versus height of inclined plane to minimum film thickness ratio, $\delta^{*}$, for pads with different $B / L$ values, and $h_{2}=10 \mu \mathrm{m}$.

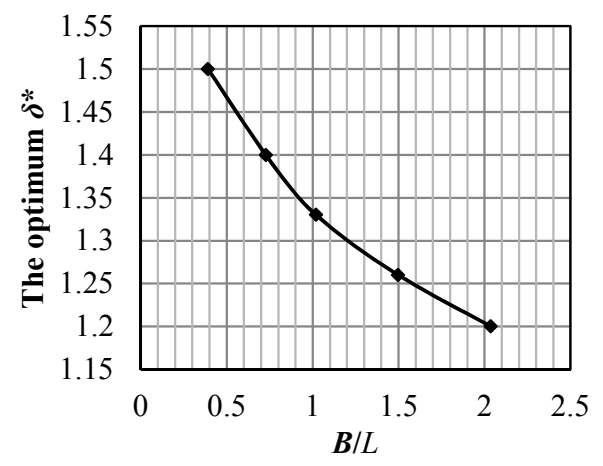

Fig. 9. The optimum $\delta^{*}$ versus pad width-to-length ratio $B / L$.

The optimum pivot position of tilting-pad thrust bearing can be determined according to the optimum $\delta^{*}$. The result is summarized in Figure 13. It shows that the optimum $R_{s}^{*}$ ranges from 0.47 to 0.57 when $B / L$ increases from 0.39 to 2.04; the optimum $\theta_{s}^{*}$ ranges from 0.567 to
0.577 , and it reduces slightly with $B / L$ when $B / L$ is less than 1.5 , while remains unchanged when $B / L$ is more than 1.5 .

\subsubsection{Check computation of the minimum film thickness}

Figure 14 presents the obtained non-dimensional load carrying capacity $F_{z}^{*}$ versus pad width-to-length ratio $B / L$. It shows that $F_{z}^{*}$ increases as $B / L$ increases. The result is compared with that of rectangular pad obtained by Jakobson [29] using finite difference method. $F_{z}{ }^{*}$ is larger than that obtained by Jakobson. It indicates that sector pads provide greater load carrying capacity than rectangular ones and the difference increases as $B / L$ increases. This is because as $B / L$ increases, pressure center in a sector pad moves towards the outside radius, where the fluid velocity is much larger than that at the middle radius. Thus larger pressure and greater load are gained in a sector pad than those in a rectangular pad. It can also be seen that the slope of the load curve for small values of $B / L$ is larger than 


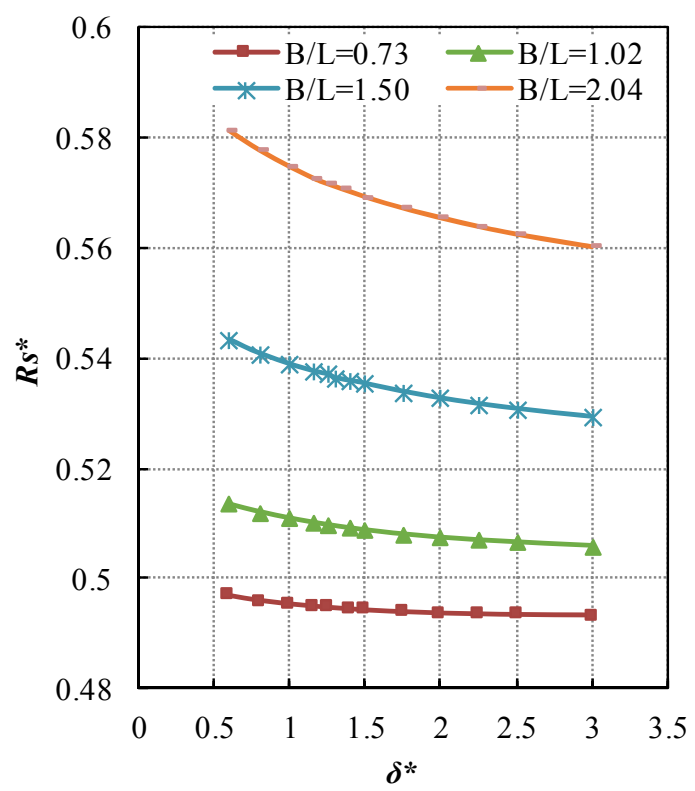

Fig. 10. Non-dimensional radius of pivot, $R_{s}^{*}$, versus $\delta^{*}$ for pads with different $B / L$ values, and $h_{2}=10 \mu \mathrm{m}$.

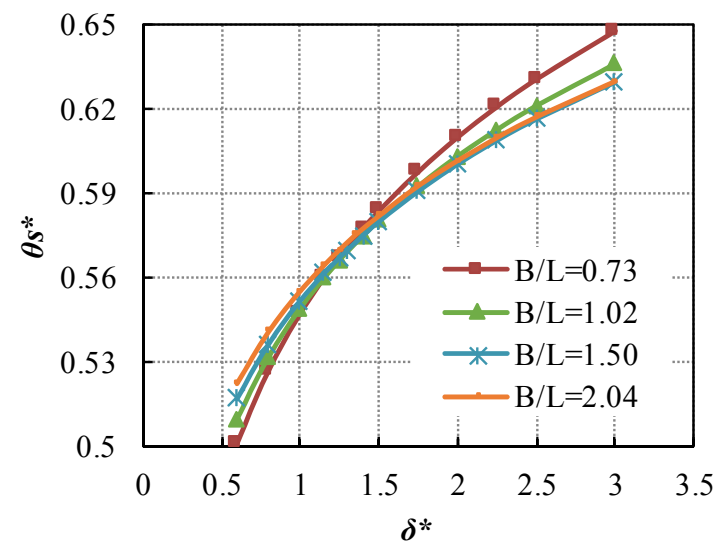

Fig. 11. Non-dimensional angle of pivot, $\theta_{s}^{*}$, versus $\delta^{*}$, for pads with different $B / L$ values, and $h_{2}=10 \mu \mathrm{m}$.

that for large values of $B / L$. This is because the pressure buildup is more affected by side leakage for small $B / L$ values.

The minimum film thickness $h_{2}$ can be calculated from $F_{z}^{*}$.As illustrated in Figure $14, B / L$ is the main influence factor on the non-dimensional load carrying capacity $F_{z}{ }^{*}$ for a constant $\delta^{*}$. This means for a certain $B / L$ value, $F_{z}{ }^{*}$ is definite. Then the minimum film thickness $h_{2}$, an important criterion of lubrication mode, can be calculated from equation (12):

$$
h_{2}=\sqrt{\frac{F_{z}{ }^{*} \cdot n \mu U L^{2} B}{F_{z}}} .
$$

In order to ensure that the bearings operate under hydrodynamic lubrication, the surface roughness values of

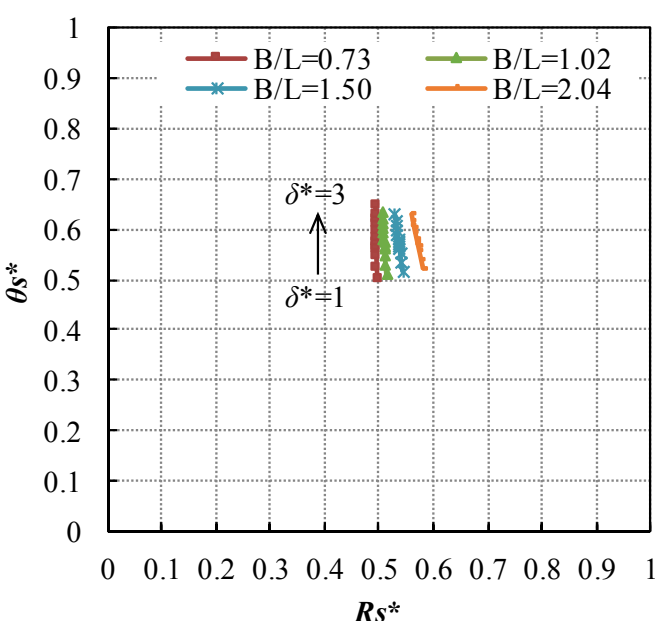

Fig. 12. Change of pivot position with $\delta^{*}$, for pads with different $B / L$ values, and $h_{2}=10 \mu \mathrm{m}$.

the thrust pad and the thrust ring should satisfy the following formula:

$$
\sqrt{R_{q, p}^{2}+R_{q, r}^{2}} \leq \frac{h_{2}}{S}
$$

where $R_{q, p}$ refers to rms (root mean square) surface roughness of the pad surface, $R_{q, r}$ refers to rms surface roughness of the ring surface, $S$ refers to the minimum film thickness to surface roughness ratio corresponding to the change of bearing lubrication regime from mixed lubrication to hydrodynamic lubrication, which can be obtained from experiments.

If the surface roughness values could not meet the design requirements, the bearing parameters should be corrected according to equations (12) and (13).

\section{Experimental test}

In order to test the lubrication properties of waterlubricated tilting-pad thrust bearing and verify the theoretical calculation method, a water-lubricated bearing test rig is built, a thrust bearing is designed according to the numerical results and the friction coefficient and water film thickness of the tilting-pad thrust bearing under different load and speed are tested.

\subsection{Water-lubricated bearing test rig}

Figure 15 shows the water-lubricated bearing test rig. The test rig includes mechanical part and test system. Figure 16 shows schematic of the test rig. Shaft 4 is driven by servo motor and the shaft speed can be adjusted by motor driver. Shaft 4 is supported by rolling bearing 3 with high precision and its rotation accuracy reaches $2 \mu \mathrm{m}$. Test shaft 5 is fitted with shaft 4 through a conical surface and fixed by bolts. In order to test the friction coefficient of water-lubricated bearing directly, the test thrust bearing 8 is mounted on the ball seat 11 through a thrust ball bearing and a journal 

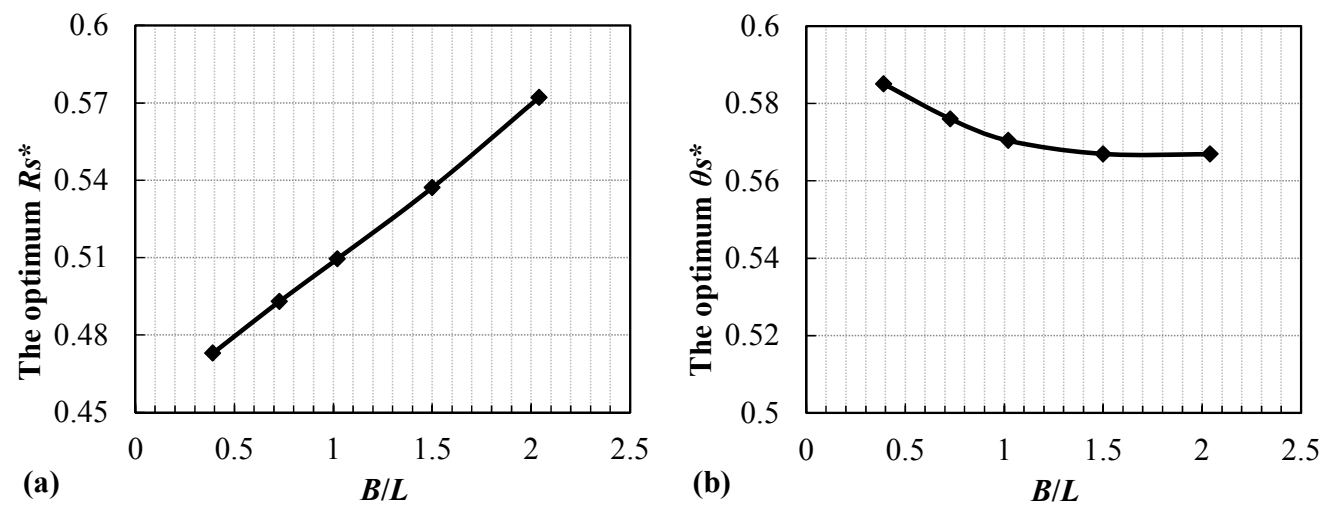

Fig. 13. Optimum position of pivot in tilting pad thrust bearing for different $B / L$.

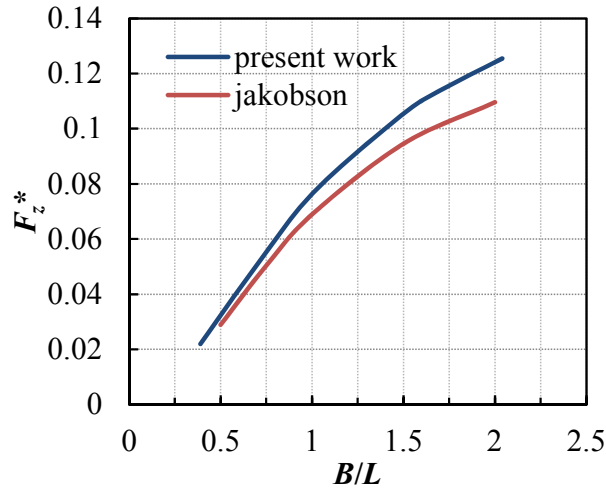

Fig. 14. Non-dimensional load carrying capacity for different pad width-to-length ratio.

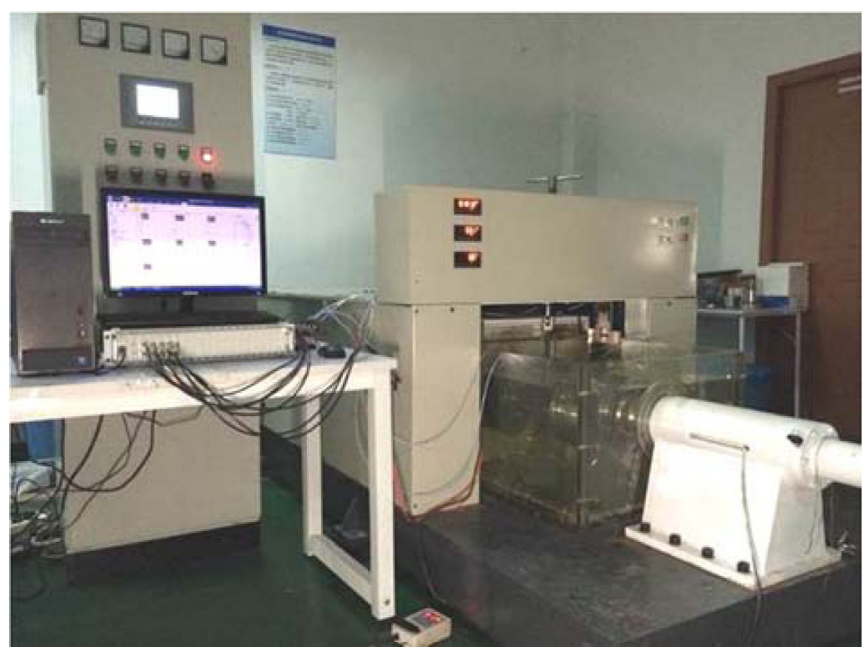

Fig. 15. Water-lubricated bearing test rig.

bearing component 10 . The test bearing is connected to a force sensor 7 via a friction torque measuring rod 6 , which can be seen more clearly in Figure 17. The error of friction torque of test bearing is mainly caused by the static friction force of component 10 . When the friction torque of test bearing is less than the friction torque of component 10 , the measurement is incorrect. In this case, an initial value of force sensor 7 is set artificially by tightening the line. So the

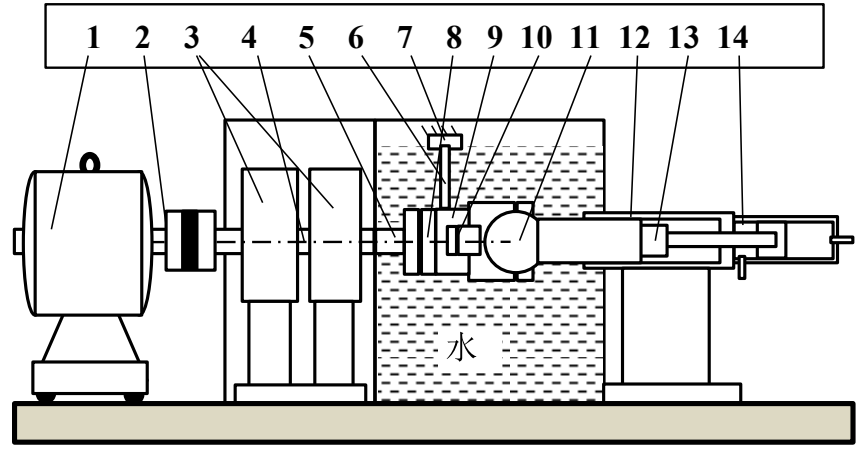

Fig. 16. Schematic of water-lubricated bearing test rig: 1-servo motor; 2-coupling; 3-rolling bearings; 4-shaft; 5 -test shaft; 6measuring rod for friction torque; 7 -friction torque force sensor; 8thrust bearing; 9-thrust bearing pedestal; 10-thrust ball bearing and journal bearing; 11-ball seat; 12-guide rail; 13-force sensor; 14-air cylinder.

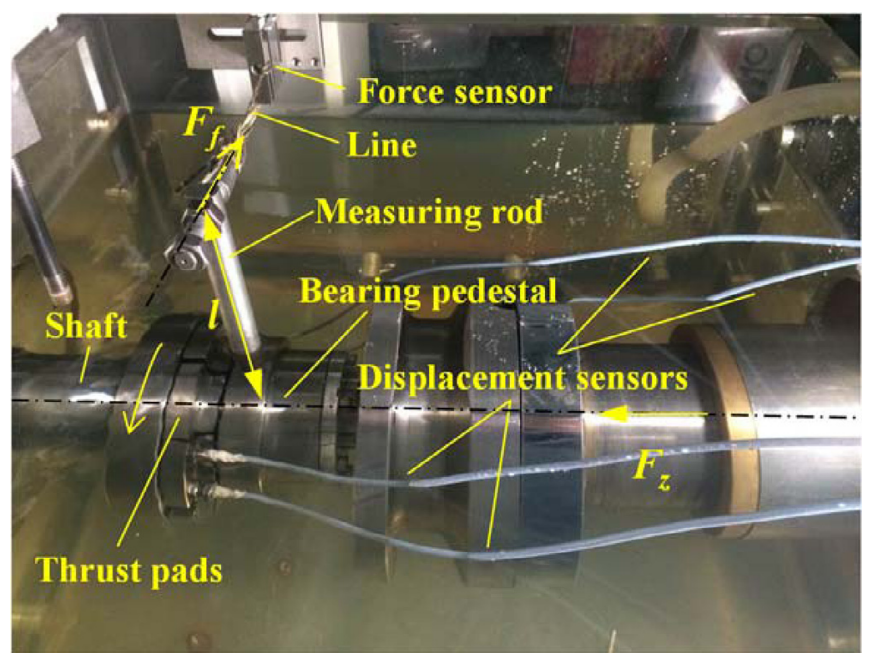

Fig. 17. Measurement part of water-lubricated thrust bearing test rig.

measured friction torque may be a little larger. But the variation of the friction torque is accurate. The thrust bearing is loaded by pneumatic loading method. The thrust load is measured by force sensor 13 . 


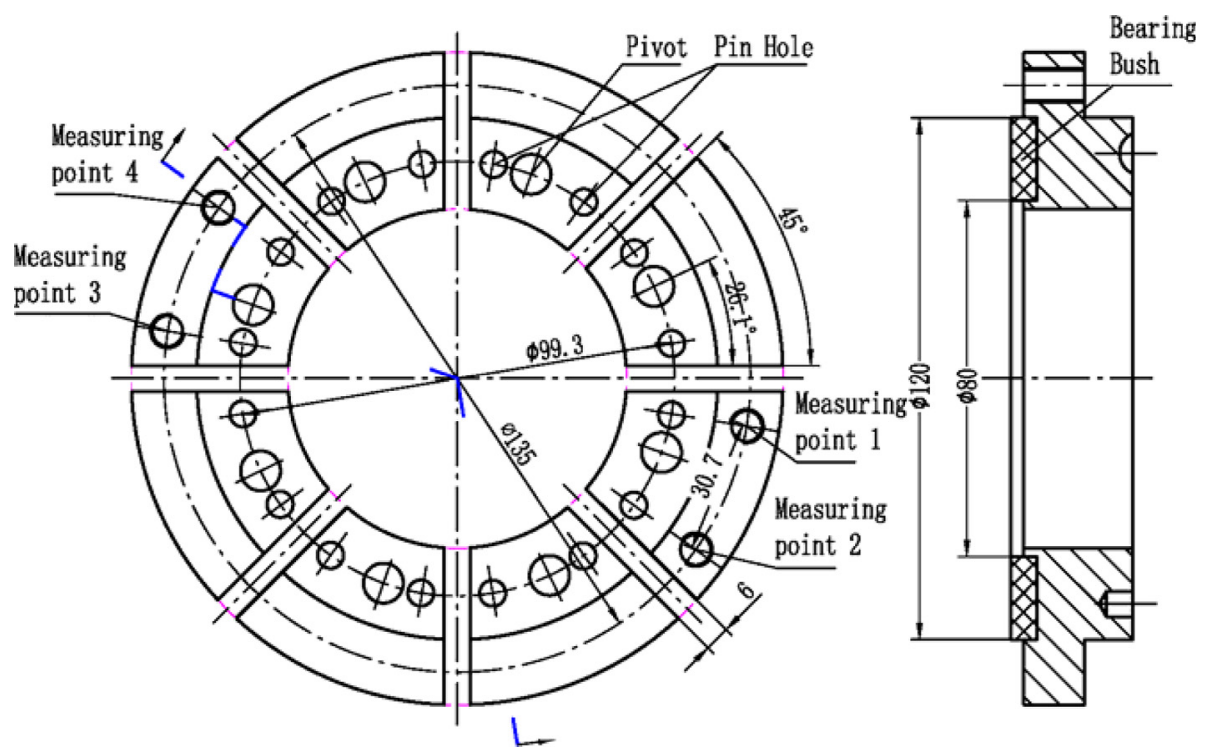

Fig. 18. Structure of test bearing.

To determine whether the water-lubricated bearing operates under hydrodynamic lubrication regime, the direct criterion is the minimum film thickness. When the minimum film thickness exceeds the minimum film thickness under mixed lubrication regime and reaches a certain value, the bearing will operated under hydrodynamic lubrication regime. The experiment aims to find the minimum film thickness to surface roughness ratio corresponding to the change of bearing lubrication regime from mixed lubrication to hydrodynamic lubrication. Four displacement sensors are used to measure the film thicknesses.

The test shaft is made of $42 \mathrm{CrMo}$ and it is electroplated with a layer of nickel for rust protection. The outer diameter of the test thrust plate is $150 \mathrm{~mm}$ and the surface roughness is $\mathrm{Ra} 0.3 \mu \mathrm{m}$.

The test system includes eddy current displacement sensors, force sensors, a data acquisition and analysis equipment and a computer. The performance parameters of the eddy current displacement sensor are: probe diameter $5 \mathrm{~mm}$, starting position $0.5 \pm 0.1 \mathrm{~mm}$, range $1 \mathrm{~mm}$, resolution $0.1 \mu \mathrm{m}$, temperature drift $\leq 0.05 \% /{ }^{\circ} \mathrm{C}$ FS. Before the test, the eddy current sensor and the force sensor were calibrated by the manufacturer to ensure the measurement accuracy. As there is a problem of magnetic run out of the thrust collar when using the eddy current displacement sensor to test the change of film thickness, chebyshev low-pass filter is used to handle the signal.

\subsection{Test bearing and test method}

Figure 18 shows structure of the test thrust bearing. It is composed of bushing and backing plate. The backing plate is made of stainless steel. The bearing bushing uses Polytetrafluoroethylene (PTFE) as base material and is reinforced with Polyetheretherketone (PEEK) and carbon fiber. The elastic modulus of the composite bush is about $1300 \mathrm{MPa}$. The pads of the test bearing are cut from a circular ring with a flat thrust surface, and the supporting

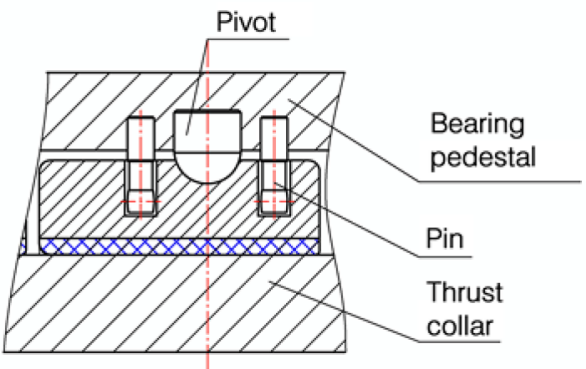

Fig. 19. Installation diagram of thrust pad.

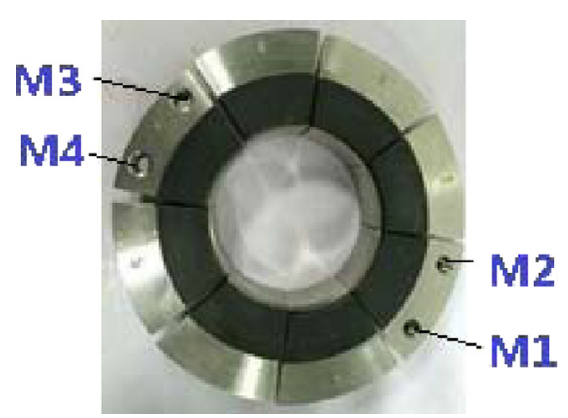

Fig. 20. Water-lubricated thrust bearing.

ball surface and groove surface are processed and formed in one step. The inner diameter of the bearing pad is $80 \mathrm{~mm}$, the outer diameter is $120 \mathrm{~mm}$, and the surface roughness is $1.7-2.2 \mu \mathrm{m}$. There are 8 pads and the water groove is designed as straight groove for easy machining. The width of water groove is $6 \mathrm{~mm}$, so the pad width-to-length ratio is 0.6 . The optimum non-dimensional pivot position is $R_{s}^{*}=0.483, \theta_{s}^{*}=0.58$ according to Figure 13 , so the supporting position is designed as $R_{s}=49.65 \mathrm{~mm}, \theta_{s}=$ $26.1^{\circ}$. The thrust pad is supported by a ball and limited by two pins as shown in Figure 19. Figure 20 shows the test water-lubricated thrust bearing. 


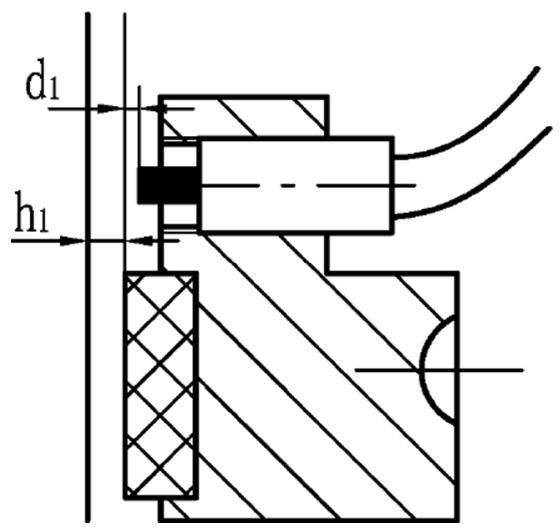

Fig. 21. Measurement of thrust bearing film thickness.

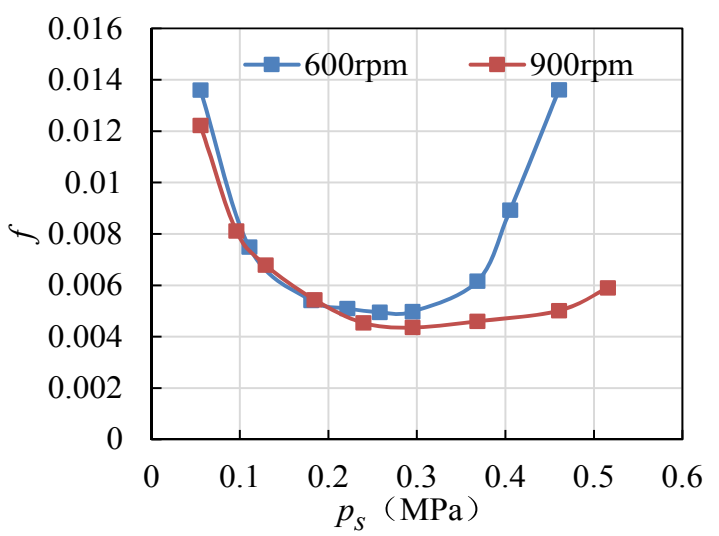

Fig. 22. Friction coefficient versus specific pressure for $N=600$ and $900 \mathrm{rpm}$.

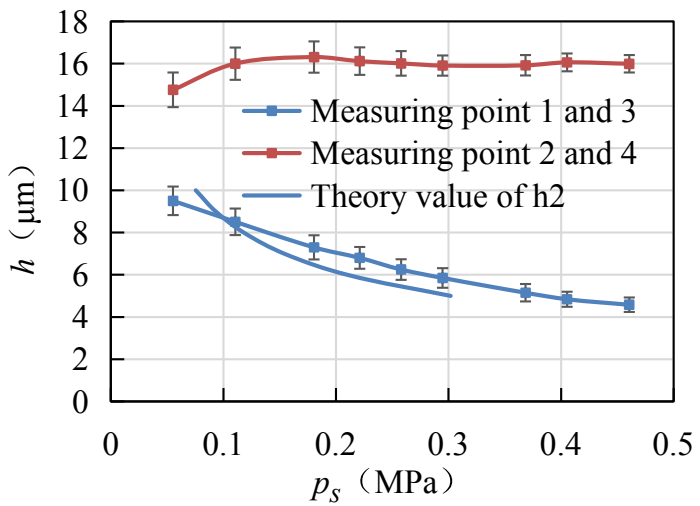

Fig. 23. Film thickness versus specific pressure for $N=600 \mathrm{rpm}$.

As the thrust bearing is small, the displacement sensors can not be installed at the bearing bush to measure the water film thickness directly. So the sensors are installed outside the bearing bush. The mounting position of displacement sensors is shown in Figures 18 and 20. The water film thicknesses on two pads are measured and the average value of film thicknesses at the same location is used to eliminate the effect of shaft inclination. Figure 21

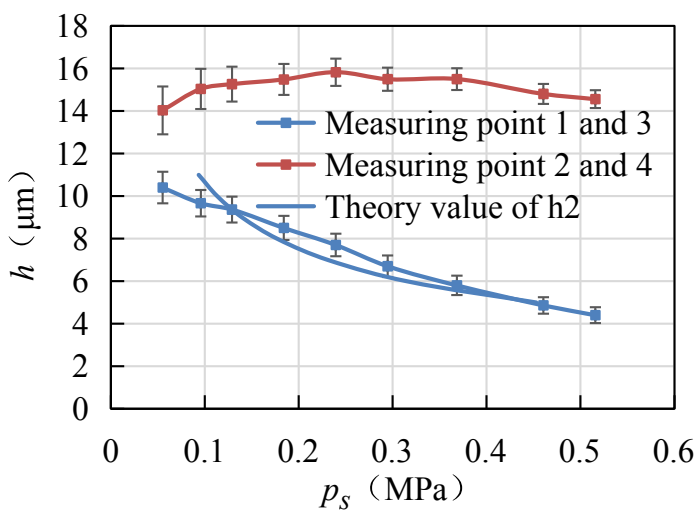

Fig. 24. Film thickness versus specific pressure for $N=900 \mathrm{rpm}$.

shows test method of the water film thickness. It can be seen that the measured data of sensor is:

$$
X_{1}=d_{1}+h_{1},
$$

where $d_{1}$ is the distance from the sensor probe to the surface of bearing bush. $d_{1}$ is constant as the sensor is fixed on the bearing backing plate. Therefore, before the test the position of sensors need to be calibrated and $d_{1}$ is measured.

The measurement principle of bearing friction coefficient is introduced in Ch. 4.1. The friction coefficient can be calculated using the following equation:

$$
F_{f} l=f \cdot F_{z} R_{m},
$$

where $F_{f}$ is the measured value of force sensor $7, l$ is the distance from the shaft axis to the sensor measuring line, $F_{z}$ is the load (see Fig. 17).

\subsection{Results and discussion}

4.3.1 Effect of load on bearing friction coefficient and film thickness

Figure 22 shows the change of friction coefficient of the water-lubricated thrust bearing with load for $N=600$ and $900 \mathrm{rpm}$. When the load is small, the bearing operates under hydrodynamic lubrication regime and the bearing friction is mainly the liquid shear force, which change little with load. So, the friction coefficient decreases when load increases. When load continues to increases, asperity contact appears and the bearing friction includes the liquid shear force and the asperity contact friction. The larger the load, the smaller the film thickness and the greater the asperity contact frictional force. When the increase of friction is larger than the increase of load, the friction coefficient increases.

Figures 23 and 24 present the variation of water film thickness with load for $N=600$ and $900 \mathrm{rpm}$, respectively. The lines with marks show the mean film thicknesses at measuring point 1 and 3 or 2 and 4 . The error bars present the differences between film thicknesses measured at measuring point 1 and 3 or 2 and 4 . The solid line refers to the theoretical minimum film thickness. It can be seen that when the load increases, the minimum water film thickness decreases and the pad inclined angle increases. As 


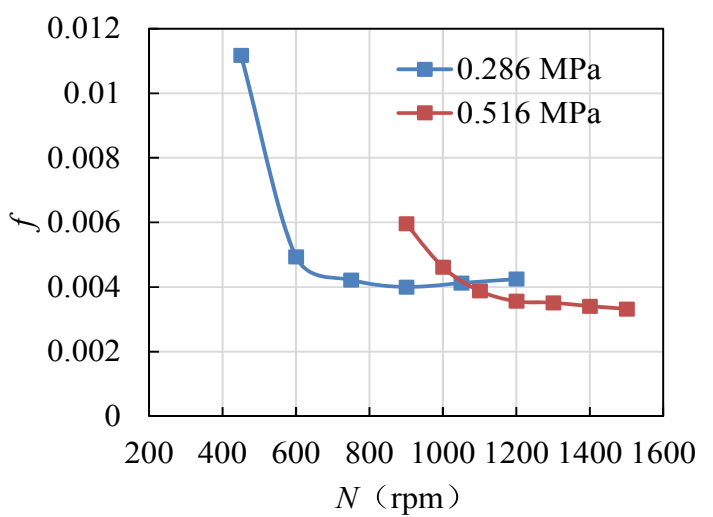

Fig. 25. Friction coefficient versus rotational speed for $p_{s}=$ 0.286 and $0.516 \mathrm{MPa}$.

the displacement sensor is not installed at the bearing bush, so there is deviation between measured value and theoretical value of film thickness, but the trend is the same. According to the film thickness of measuring point 1 and measuring point 2 , the calculated inclined angle is about $0.01^{\circ}-0.02^{\circ}$, which is in agreement with the theoretical value calculated by equation (11) in the order of magnitude. From the change of error bars, it can be seen that as load increases the difference between film thicknesses at the two pads decreases. This might be due to the bearing inclination. The inclination of bearing axis decreases as load increases.

From Figures 22 and 23, it can be seen that at $600 \mathrm{rpm}$ the friction coefficient begins to increase when the specific pressure is $0.3 \mathrm{MPa}$, and the minimum water film thickness is $5.85 \mu \mathrm{m}$. From Figures 22 and 24, it can be seen that at $900 \mathrm{rpm}$ the friction coefficient begins to increase when the specific pressure is $0.37 \mathrm{MPa}$, and the minimum water film thickness is $5.8 \mu \mathrm{m}$. The increasing friction coefficient indicates that the bearing has been in mixed lubrication regime, so the minimum film thickness corresponding to the change of bearing lubrication regime from mixed lubrication to hydrodynamic lubrication is larger than $5.8 \mu \mathrm{m}$.

\subsection{Effect of rotational speed on bearing friction coefficient and film thickness}

Figure 25 shows the change of friction coefficient with rotational speed for $p_{s}=0.286$ and $0.516 \mathrm{MPa}$. For small rotational speed, the water film thickness is small and there is asperity contact, so the friction coefficient is large. As rotational speed increases, water film thickness increases and the bearing friction coefficient decreases. When rotational speed increases to a certain value, hydrodynamic lubrication forms and the bearing friction is mainly liquid shear force, which increases with rotational speed, so the bearing friction coefficient increases when rotational speed continues to increase.

Figures 26 and 27 present the change of water film thickness with rotational speed for $p_{s}=0.286$ and $0.516 \mathrm{MPa}$. The lines with marks show the mean film thicknesses at measuring point 1 and 3 or 2 and 4 . The error bars present the differences between film thicknesses

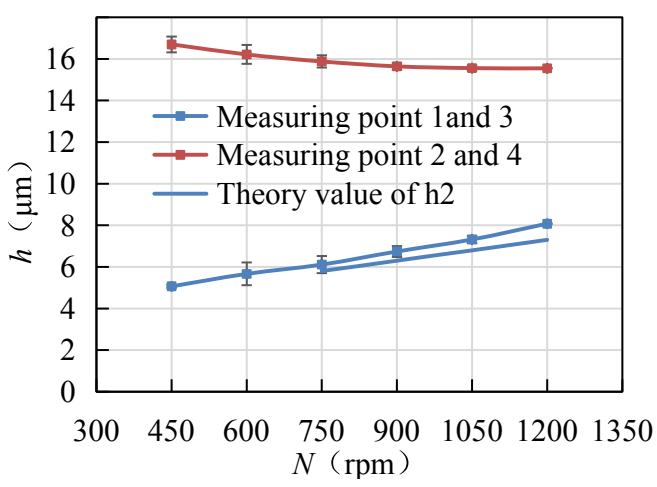

Fig. 26. Film thickness versus rotational speed for $p_{s}=0.286$ MPa.

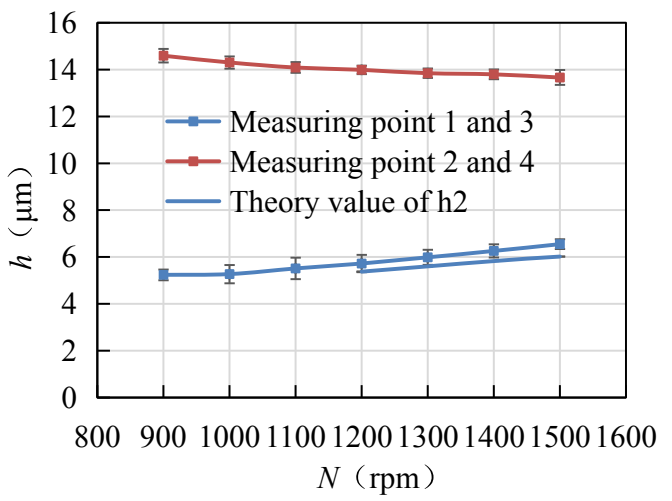

Fig. 27. Film thickness versus rotational speed for $p_{s}=0.516$ $\mathrm{MPa}$.

measured at measuring point 1 and 3 or 2 and 4 . The solid line shows the change of theoretical minimum film thickness. When the load is constant, the minimum water film thickness increases and the pad inclined angle decreases as rotational speed increases. The measured minimum film thicknesses are slightly larger than the theoretical values, but the trend is the same. The error bar is short for $p_{s}=0.286$ and $0.516 \mathrm{MPa}$ and its change rule with rotational speed is not clear.

From Figures 25 and 26, it can be seen that for $p_{s}=0.286 \mathrm{MPa}$ the bearing friction coefficient begins to increase when the rotational speed is about $900 \mathrm{rpm}$, and the minimum water film thickness is $7.2 \mu \mathrm{m}$. When $p_{s}$ is $0.516 \mathrm{MPa}$, the friction coefficient does not show the rise when rotational speed is less than $1500 \mathrm{rpm}$, and the minimum water film thickness is $6.5 \mu \mathrm{m}$ at $N=1500 \mathrm{rpm}$ as shown in Figure 27. It indicates that the minimum water film thickness is about $7.2 \mu \mathrm{m}$ when the bearing lubrication regime changes from mixed lubrication to hydrodynamic lubrication. Since the surface roughness of the test bearing is $1.7-2.2 \mu \mathrm{m}$, the minimum film thickness to surface roughness ratio is about 3.6.

\section{Conclusions}

In this research, the hydrodynamic lubrication model of the water-lubricated tilting-pad thrust bearing with sector 
pads and straight radial grooves has been fully studied using CFD method. The effects of pad inclined angle, pivot position and pad width-to-length ratio on lubrication properties are discussed. Experiments are carried out to study the lubrication performance of tilting-pad thrust bearing and the correctness of theoretical method. The main conclusions are drawn as follows:

- the optimum pivot position of tilting-pad thrust bearing can be chosen from Figure 14 according to $B / L$. The optimum $R_{s}{ }^{*}$ ranges from 0.47 to 0.57 when $B / L$ increases from 0.39 to 2.04 ; the optimum $\theta_{s}^{*}$ ranges from 0.567 to 0.577 , and it reduces slightly with $B / L$ when $B / L$ is less than 1.5 , while remains unchanged when $B / L$ is more than 1.5 ;

- the non-dimensional load carrying capacity of tilting-pad thrust bearing can be found in Figure 14. It varies from 0.022 to 0.125 as $B / L$ increases from 0.39 to 2.04 . Compared with rectangular pad, sector pad provides greater load carrying capacity and the difference increases as $B / L$ increases;

- the minimum film thickness should be checked using equations (12) and (13) in order to ensure that the bearings operate under hydrodynamic lubrication. Experiments showed that the minimum film thickness to surface roughness ratio corresponding to the change of bearing lubrication regime from mixed lubrication to hydrodynamic lubrication in equation (13) is about 3.6;

- the measured film thicknesses are in accordance with the theoretical values, which validates the theoretical method.

The bush deformation and installation error of pads also influence the lubrication and load carrying properties. They have not been considered in this work. We'll study their effects in the future work.

\section{Nomenclature}

\section{$B \quad$ pad width $=R_{2}-R_{1}$}

$\mathrm{B} / \mathrm{L} \quad$ pad width-to-length ratio

$F_{z_{*}} \quad$ load carrying capacity of a thrust bearing

$F_{z} \quad$ non-dimensional load carrying capacity

$f \quad$ friction coefficient $=M_{\mathrm{f} r} /\left(F_{z} R_{m}\right)$

$h_{1}, h_{2} \quad$ film thicknesses at inlet and outlet of clearance in middle radius

$h_{g} \quad$ groove depth

$k \quad$ pad ratio $=\theta_{p} /\left(\theta_{p}+\theta_{g}\right)$

$L \quad$ pad length (see Fig. 1), $L=2 \pi R_{m} k / n$

$L_{\mathrm{g}} \quad$ groove length

$M_{\mathrm{fr}_{*}} \quad$ friction torque on the thrust ring

$M_{\mathrm{fr}}{ }^{*}$ non-dimensional friction torque= $M_{f r} h_{2}\left(n \mu U_{m} L B R_{m}\right)$

$\mathrm{N} \quad$ rotational speed

$n \quad$ number of pads

$p \quad$ pressure

$p_{s_{*}} \quad$ specific pressure $=F_{z} /(n B L)$

$p^{*} \quad$ non-dimensional pressure $=p h_{2}^{2} /(\mu U L)$

$R_{1}, R_{m}, R_{2}$ bearing inner, middle and outer radius

$R_{s_{*}} \quad$ radius of pivot

$R_{s} \quad$ non-dimensional radius of pivot $=\left(R_{s}-R_{1}\right)$ B $\rho$

linear velocity of the thrust collar $=2 \pi N R$, $U_{m}=2 \pi N R_{m}$ velocity vector pad angle, groove angle angle of pivot non-dimensional angle of pivot $=\theta_{s} / \theta_{p}$ height of inclined plane $=h_{1}-h_{2}$ non-dimensional height of inclined plane $=\delta /$ $h_{2}$ pad inclined angle fluid dynamic viscosity fluid density

Acknowledgements. The authors thank the editor and reviewers for their helpful comments and suggestions. The financial support from the National Natural Science Foundation of China (Grant No.11672178, Grant No.51705310 and Grant No.2017M611555) is also gratefully acknowledged.

\section{References}

[1] S.B. Glavatskih, Steady state performance characteristics of a tilting pad thrust bearing, J Tribol 123 (2001) 608

[2] J. Wang, F. Yan, Q. Xue, Tribological behavior of PTFE sliding against steel in sea water, Wear 267 (2009) 1634-1641

[3] J.H. Jia, J.J. Lu, H.D. Zhou, J.M. Chen, Tribological behavior of Ni-based composite under distilled water lubrication, Mater Sci Eng A Struct Mater Prop Microstruct Process 381 (2004) 80-85

[4] W. Huang, Y. Xu, Y. Zheng, X. Wang, The tribological performance of $\mathrm{Ti}(\mathrm{C}, \mathrm{N})$-based cermet sliding against Si3N4 in water, Wear 270 (2011) 682-687

[5] H. Unal, A. Mimaroglu, Friction and wear characteristics of PEEK and its composite under water lubrication, J Reinf Plast Compos 25 (2006) 1659-1667

[6] J. Jia, J. Chen, H. Zhou, J. Wang, H. Zhou, Friction and wear properties of bronze-graphite composite under water lubrication, Tribol Int 37 (2004) 423-429

[7] Y.D. Tridimas, D.R. Allanson, N.H. Woolley, D.L. Cabrera, Film pressure distribution in water-lubricated rubber journal bearings, Proc Inst Mech Eng Part J: J Eng Tribol 219 (2005) 125-132

[8] N. Wang, Q. Meng, P. Wang, T. Geng, X. Yuan, Experimental research on film pressure distribution of water-lubricated rubber bearing with multiaxial grooves, J Fluids Eng 135 (2013) 084501

[9] R. Pai, R.S. Pai, Stability of four-axial and six-axial grooved water-lubricated journal bearings under dynamic load, Proc Inst Mech Eng Part J: J Eng Tribol 222 (2008) 683-691

[10] B.C. Majumdar, R. Pai, D.J. Hargreaves, Analysis of waterlubricated journal bearings with multiple axial grooves, Proc Inst Mech Eng Part J: J Eng Tribol 218 (2004) 135-146

[11] B.D. Heberley, Advances in hybrid water-lubricated journal bearings for use in ocean vessels, Massachusetts Institute of Technology, Ann Arbor, USA, 2013.

[12] G.Y. Gao, Z.W. Yin, D. Jiang, X.L. Zhang, Numerical analysis of plain journal bearing under hydrodynamic lubrication by water, Tribol Int 75 (2014) 31-38

[13] W. Litwin, Marine water lubricated stern tube bearings calculations and measurements of heavy loaded bearings, in: Proceedings of the STLE/ASME International Joint 
Tribology Conference IJTC2008, Miami, Florida, USA, 2008.

[14] W. Litwin, Experimental investigation on marine main shaft bearings with reduced length to diameter ratio, in: Proceedings of the STLE/ASME 2010 International Joint Tribology Conference IJTC2010, San Francisco, California, USA 2010.

[15] W. Litwin, Experimental research on water lubricated three layer sliding bearing with lubrication grooves in the upper part of the bush and its comparison with a rubber bearing, Tribol Int 82 (2015) 153-161

[16] W. Litwin, C. Dymarski, Experimental research on waterlubricated marine stern tube bearings in conditions of improper lubrication and cooling causing rapid bush wear, Tribol Int 95 (2016) 449-455

[17] X. Zhang, X. Wang, X. Li, X. Chu, Study on influence factors of bearing capacity of water-lubricated thrust bearing and its improving methods, in Advanced Mechanical Design, 2012

[18] X. Zhang, Z. Yin, D. Jiang, G. Gao, The design of hydrodynamic water-lubricated step thrust bearings using CFD method, Mech Ind 15 (2014) 197-206

[19] S. Nakano, T. Kishibe, T. Inoue, H. Shiraiwa, An advanced microturbine system with water-lubricated bearings, Int J Rotating Mach (2009) 1-12

[20] K. Inoue, K. Deguchi, K. Okude, R. Fujimoto, Development of the water-lubricated thrust bearing of the hydraulic turbine generator, in: IOP Conference Series: Earth and Environmental Science, Vol. 15, 2012072022 p.

[21] R. Pai, D.J. Hargreaves, Water lubricated bearings, in: Green Tribology, Springer, Berlin Heidelberg, 2012

[22] X. Wang, K. Koji, A. Koshi, A. Kohji, The effect of laser texturing of $\mathrm{SiC}$ surface on the critical load for the transition of water lubrication mode from hydrodynamic to mixed, Tribol Int 34 (2001) 703-711

[23] X.L. Wang, K. Kato, K. Adachi, K. Aizawa, Loads carrying capacity map for the surface texture design of $\mathrm{SiC}$ thrust bearing sliding in water, Tribol Int 36 (2003) 189-197

[24] D.L. Cabrera, N.H. Woolley, D.R. Allanson, Y.D. Tridimas, Film pressure distribution in water-lubricated rubber journal bearings, Proc Inst Mech Eng Part J: J Eng Tribol 219 (2005) 125-132

[25] X.L. Wu, Lubrication design handbook, Chemical Industry Press, Beijing, 2006

[26] ANSYS, ANSYS FLUENT, version 14.0: user manual, ANSYS Inc, Canonsburg, USA, 2011

[27] P.J. Zwart, A.G. Gerber, T. Belamri, A two-phase flow model for predicting cavitation dynamics, in Fifth International Conference on Multiphase Flow, Yokohama, Japan, 2004

[28] G. Zhou, J. Ma, Y. Quan, Fluid lubrication theory, Zhejiang University, Hangzhou, 1990

[29] B. Jakobsen, L. Floberg, The rectangular plane pad bearing, Gumpert, 1958

Cite this article as: X. Zhang, G. Gao, Z. Yin, Y. Wang, C. Gao, Numerical analysis and experimental research on load carrying capacity of water-lubricated tilting-pad thrust bearings, Mechanics \& Industry 19, 201 (2018) 